\title{
TonB-dependent transporters expressed by Neisseria gonorrhoeae
}

\section{Cynthia Nau Cornelissen* and Aimee Hollander}

Department of Microbiology, Virginia Commonwealth University Medical Center, Richmond, VA, USA

\section{Edited by:}

D. Scott Merrell, Uniformed Services

University, USA

\section{Reviewed by:}

Susan K. Buchanan, National Institutes of Health, USA

Shelley M. Payne, University of Texas, USA

Jorge Crosa, Oregon Health and

Science University, USA

*Correspondence:

Cynthia Nau Cornelissen, Department of Microbiology, Virginia

Commonwealth University Medical

Center, Richmond, VA 23298-0678,

USA.

e-mail: cncornel@vcu.edu
Neisseria gonorrhoeae causes the common sexually transmitted infection, gonorrhea. This microorganism is an obligate human pathogen, existing nowhere in nature except in association with humans. For growth and proliferation, $N$. gonorrhoeae requires iron and must acquire this nutrient from within its host. The gonococcus is well-adapted for growth in diverse niches within the human body because it expresses efficient transport systems enabling use of a diverse array of iron sources. Iron transport systems facilitating the use of transferrin, lactoferrin, and hemoglobin have two components: one TonB-dependent transporter and one lipoprotein. A single component TonB-dependent transporter also allows $N$. gonorrhoeae to avail itself of iron bound to heterologous siderophores produced by bacteria within the same ecological niche. OtherTonB-dependent transporters are encoded by the gonococcus but have not been ascribed specific functions. The best characterized iron transport system expressed by $N$. gonorrhoeae enables the use of human transferrin as a sole iron source. This review summarizes the molecular mechanisms involved in gonococcal iron acquisition from human transferrin and also reviews what is currently known about the otherTonB-dependent transport systems. No vaccine is available to prevent gonococcal infections and our options for treating this disease are compromised by the emergence of antibiotic resistance. Because iron transport systems are critical for the survival of the gonococcus in vivo, the surface-exposed components of these systems are attractive candidates for vaccine development or therapeutic intervention.

Keywords: iron, Neisseria gonorrhoeae,TonB, transferrin, xenosiderophores

\section{INTRODUCTION}

\section{NEISSERIA GONORRHOEAEAND IRON "PIRACY"}

Neisseria gonorrhoeae causes a very common, but often asymptomatic sexually transmitted infection (STI). The pathogen gains a foothold in the human host via the lower genital tract, but can ascend into the upper genital tract and beyond, disseminating through the bloodstream to the joints and skin. In rare cases, $N$. gonorrhoeae infections can even result in meningitis (Hook and Handsfield, 2008). Neonatal conjunctivitis, or ophthalmia neonatorum, results from transmission of the bacterium from an infected mother to her newborn during a vaginal delivery (Kohlhoff and Hammerschlag, 2008). These diverse manifestations require that the gonococcus thrive in a multitude of human environments, including in the blood, in semen, on mucosal surfaces, in joint fluid, and on the conjunctiva. In each niche, the pathogen must acquire all of the nutrients, including iron, that are necessary for multiplication.

Unlike most bacteria, the Neisseria species do not produce siderophores in an effort to acquire iron from the environment (West and Sparling, 1985). As well-adapted human pathogens, these bacteria instead rely entirely on iron sources obtained within the human host. Iron sources utilized by N. gonorrhoeae include transferrin (Mickelsen and Sparling, 1981) and lactoferrin (Mickelsen et al., 1982), both of which are present in micromolar concentrations in semen, the normal transmission fluid for an STI pathogen (Anderson et al., 2003). Hemoglobin (Dyer et al., 1987) and heme can also be employed by the gonococcus as sole iron sources, both of which are present periodically in the female genital tract during menses. Heterologous siderophores produced by other bacteria (xenosiderophores) are available to the gonococcus within the context of cervical or rectal infections. It is anticipated that bacteria co-inhabiting these niches produce siderophores under iron stressed conditions, and the gonococcus takes advantage of this iron-sequestering attempt by hijacking the ferric complexes en route to the producer. Aerobactin (West and Sparling, 1987) and enterobactin (Carson et al., 1999), both produced by enteric bacteria, have previously been demonstrated to support the growth of N. gonorrhoeae.

Human transferrin is a glycoprotein responsible for binding to and transporting ferric iron throughout the human body. The protein is found at highest concentrations in the serum, cerebral spinal fluid, and joint fluid, but can also be detected on mucosal membranes, particularly in inflamed tissue. Lactoferrin is found in milk, secretions, and polymorpholeukocytes. Lactoferrin is believed to be primarily responsible for iron scavenging, rather than transport, and therefore is very poorly saturated with iron in $v i v o$. By contrast, transferrin is approximately $30 \%$ saturated with iron in the serum (for a recent review, see Wally and Buchanan, 2007). Early studies of iron use by N. gonorrhoeae demonstrated that both human glycoproteins can be efficiently employed by the gonococcus as a sole source of iron. Low saturation levels did not hinder access to the bound iron. Moreover, these seminal studies demonstrated that iron is internalized, but the iron-binding protein remains intact outside of the cell. The process of iron 
removal was shown to require metabolic energy and direct contact between the glycoprotein and the bacterial cell surface (McKenna et al., 1988).

\section{TWO-COMPONENT SYSTEMS FOR IRON TRANSPORT FROM HOST PROTEINS TRANSFERRIN-IRON ACQUISITION}

The mechanism of transferrin-iron acquisition by N. gonorrhoeae has been the subject of a great deal of study. The precise details of lactoferrin and hemoglobin utilization, while expected to be similar, are less well-developed. The initial steps in characterization of the gonococcal transferrin-iron acquisition system involved the identification of two membrane proteins that bound to human transferrin (Cornelissen et al., 1992). Expression of both proteins was decreased under iron-replete conditions, consistent with a role in iron internalization. The first transferrin binding protein (Tbp) to be identified and characterized in N. gonorrhoeae was TbpA (Cornelissen et al., 1992; Figures 1 and 2). The gene that encodes this protein was sequenced and found to be similar to those encoding a family of iron transport proteins in Gram-negative bacteria known as TonB-dependent transporters. These proteins share sequence similarity primarily at their $\mathrm{N}$ - and C-termini, consistent with their shared localization and function as large outer membrane transporters (Noinaj et al., 2011). Expression of gonococcal recombinant TbpA in Escherichia coli resulted in surface presentation of the transporter and rendered E. coli capable of binding transferrin in a species-specific manner; only human transferrin was recognized (Cornelissen et al., 1993). The second Tbp (Figure 1), is encoded by the gene directly upstream of $t b p A$ (Anderson et al., 1994). TbpB contains a typical "lipobox," which is necessary for N-terminal lipid modification and outer membrane localization. The genes encoding the Tbps are preceded by a typical iron-regulatory sequence (see below) and are transcribed as an iron-repressed bicistronic operon (Ronpirin et al., 2001). However,

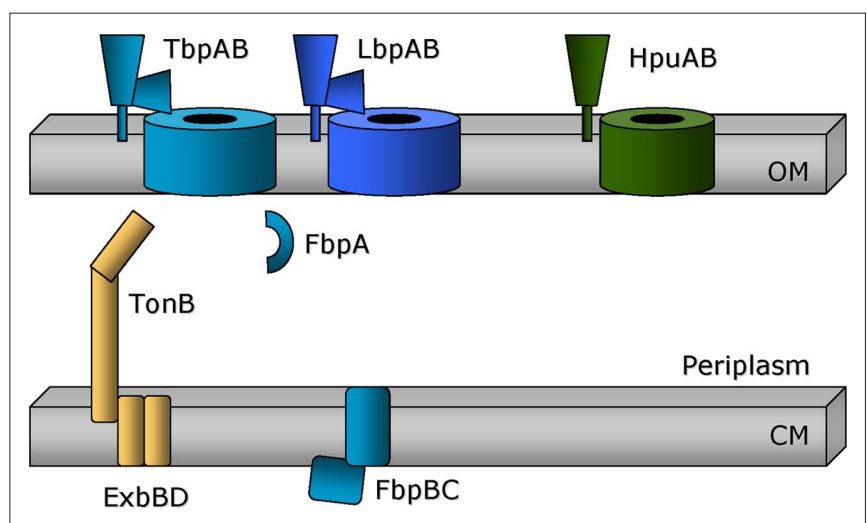

FIGURE 1 |Two component gonococcal systems for acquisition of iron from host proteins. The TonB dependent outer membrane transporters are shown as barrels traversing the outer membrane (OM). The lipid-modified companion proteins are shown tethered to the outer membrane surface. TonB, ExbB, and ExbD (gold) are depicted as attached to or imbedded within the cytoplasmic membrane (CM). The periplasmic binding protein, FbpA, is responsible for transporting iron from the outer membrane transporters, TbpA and LbpA, to the cytoplasmic membrane permease, comprised of FbpB. FbpC is the ATPase that energizes transport through FbpB. an intergenic region between $t b p B$ and $t b p A$ is capable of encoding an mRNA species with strong secondary structure potential. The observation that $t b p B$ transcripts outnumber $t b p A$ transcripts by $2: 1$ at steady state, suggests that this intervening sequence is important for maintenance of the optimum stoichiometry of the system (Ronpirin et al., 2001).

All gonococcal isolates characterized and sequenced to date possess both $t b p$ genes. However, transposon insertion mutants of $N$. gonorrhoeae were generated to test the function of these proteins in transferrin iron acquisition. Mutants lacking both proteins were incapable of growth on transferrin as a sole iron source, although growth on other iron sources was unimpaired (Anderson et al., 1994). Mutants lacking the ability to express TbpA were incapable of growth on transferrin and were unable to internalize any iron from transferrin (Cornelissen et al., 1992). By contrast, $t b p B$ mutants were still capable of growth on transferrin, but were only able to internalize approximately $50 \%$ of wild-type levels of iron from transferrin (Anderson et al., 1994). These observations led us to suggest that TbpA, the TonB-dependent transporter, was the necessary portal for iron through the outer membrane whereas $\mathrm{TbpB}$, the surface-tethered lipoprotein, was important for increased efficiency of the iron acquisition system (Figure 1).

TonB-dependent transporters share a common topology. Twelve different TonB-dependent transporters have been crystallized to date (Noinaj et al., 2011) and all share two characteristic domains: a beta-barrel comprised of 22 amphipathic beta-strands, and a globular plug domain that is folded up inside the barrel. After the first TonB-dependent transporter was crystallized (Buchanan et al., 1999), we developed a 2D model of TbpA, employing a combination of bioinformatics approaches and sequence diversity (Figure 2). Twenty-two putative amphipathic beta-strands were predicted by their similarity with the known transmembrane domains of FepA (Buchanan et al., 1999). The sequence diversity detected among five different gonococcal TbpA sequences (Cornelissen et al., 2000) was localized to regions within 11 putatively surface-exposed loops (L1-L11 in Figure 2). Likewise, three pairs of cysteine residues were localized to three extracellular loop regions (L2, L4, and L5 in Figure 2).

To begin testing the TbpA topology model, we deleted three predicted loop regions (L4, L5, and L8; Boulton et al., 2000). Mutants expressing TbpA deleted of predicted loops 4 or 5 were rendered unable to bind transferrin or use transferrin as a sole iron source. Mutants unable to express loop 8 of TbpA bound transferrin with a lower affinity but were nonetheless incapable of transferrin iron utilization. These results suggested that loops 4 and 5 were critically important ligand binding regions and were therefore crucial for transferrin-iron internalization. The phenotype of the loop 8 deletion mutant suggested that high affinity interactions between TbpA and transferrin were necessary for iron internalization and perhaps for iron removal from transferrin. We subsequently cloned regions encoding several predicted, surface-exposed loops of gonococcal TbpA (Masri and Cornelissen, 2002). Each predicted loop region was expressed as a fusion with a heterologous cellulose binding protein. The recombinant proteins were tested for their ability to bind human transferrin and amazingly two loop regions retained this ligand binding capability. Recombinant proteins comprised of predicted loop 5 or loops 4 and 5 together specifically bound 


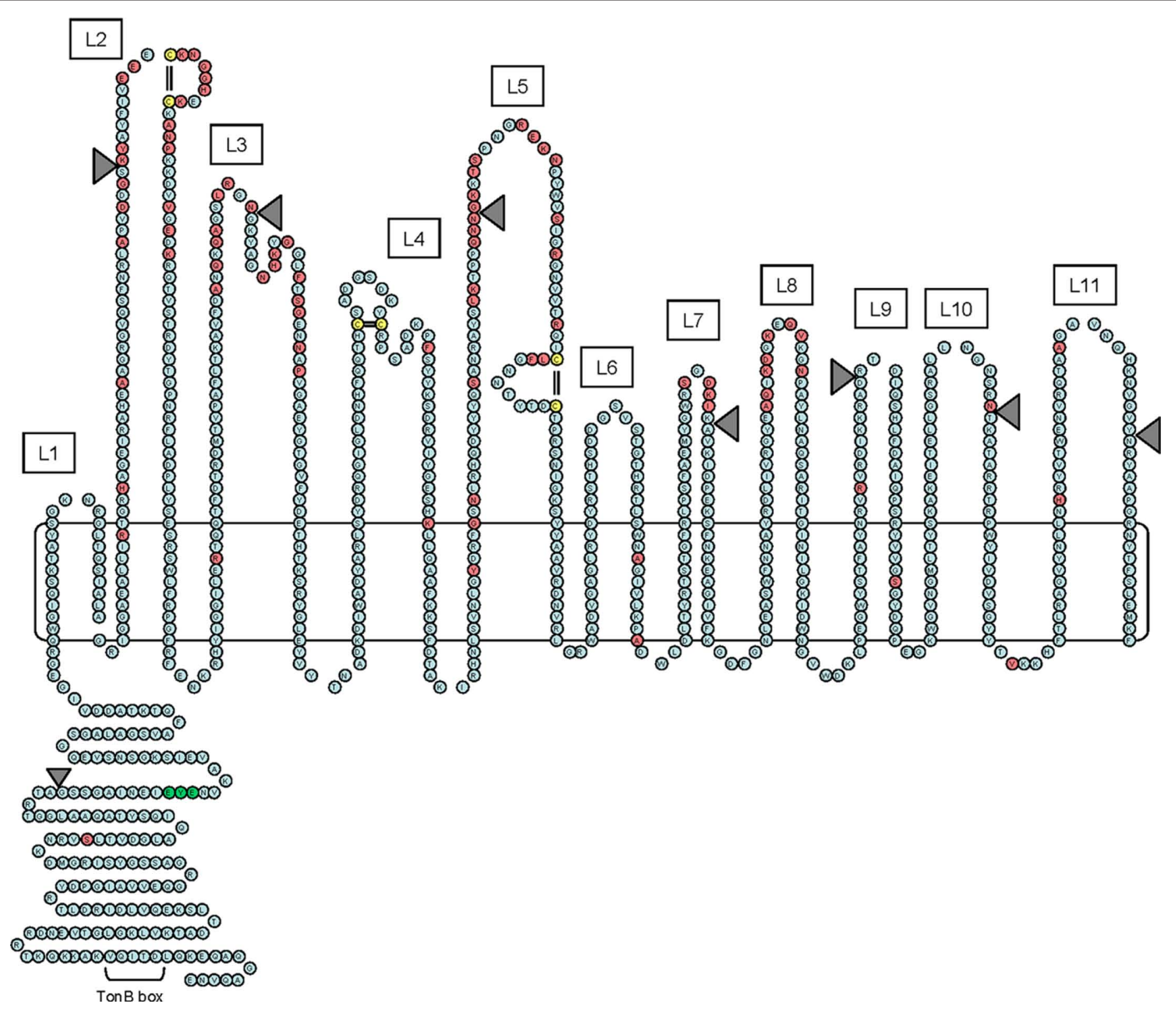

FIGURE 2 | Hypothetical two-dimensional topology model of gonococcal TbpA. The sequence shown is of the TbpA protein from gonococcal strain FA19. The model includes an amino-terminal plug domain of 161 residues, 11 surface-exposed loops (numbered L1-L11), and 22 transmembrane betastrands. Residues highlighted in red are those that are divergent among a panel of five gonococcal TbpA sequences. Cysteine residues are highlighted in yellow. The EYE residues that were mutated within the plug domain are highlighted in green. The position of HA epitopes inserted into putative loops and the plug domain are shown as gray-shaded triangles. The TonB-box region within the plug domain is also indicated. human transferrin, even out of context of the intact outer membrane protein. These data supported our earlier findings that loops 4 and 5 contained critical binding determinants.

We developed polyclonal antibodies against the over-expressed loop regions, and found that loops 2 and 5 (Figure 2) were indeed surface exposed on intact gonococci (Masri, 2003). We also determined that antibodies generated against the plug region of TbpA were also capable of specific binding to the cell surface of gonococci. However, none of these antibodies blocked transferrin binding in liquid phase binding assays. We propose that the binding affinity of human transferrin to TbpA $\left(K_{\mathrm{d}} \sim 10 \mathrm{nM}\right)$ is greater than that of these epitope-specific antibodies and therefore we detected no inhibition. Competition between the natural ligand (human transferrin) and epitope-specific antibodies also may have led to our inability to detect bactericidal activity by these antibodies in the presence of human complement. Loop 5-specific antibodies were bactericidal when baby rabbit complement was employed, suggesting that the non-human species of transferrin present in the rabbit serum allowed for antibody binding and subsequent complement fixation and bactericidal activity (Masri, 2003).
We also employed an epitope insertion strategy to test the 2D model of TbpA topology (Figure 2). We generated fusion proteins, expressed in single copy by $N$. gonorrhoeae, in which the HA epitope was fused at various points within the TbpA protein (Yost-Daljev and Cornelissen, 2004). We inserted the HA epitope within seven putatively exposed surface loops, within two putative beta-strands, within two predicted periplasmic turns, and within the plug region. We determined that the HA epitopes localized in putative loops 2, $3,5,7$, and 10 were surface exposed, confirming the extracellular localization of these regions (Figure 2). None of the HA epitopes in periplasmic turns or beta-strands were surface accessible, consistent with the model. The HA epitope tag was accessible to the surface when expressed in the plug domain, consistent with other data suggesting surface exposure of the plug domain. Contrary to the model predictions, HA epitopes in predicted loops 9 and 11 were not surface accessible by this analysis. Overall, these data largely supported the 2D model and confirmed the extracellular location of six of the predicted loop regions. Interestingly, only two epitope insertions (L3 and $\beta 9$; Figure 2 and not shown) resulted in a defective TbpA transporter; none of the other insertions resulted 
in defects in transferrin binding or transferrin-iron utilization. The exception was the plug HA epitope insertion, which resulted in decreased binding affinity and abolished transferrin iron utilization. Another interesting class of mutants was exemplified by the L2, L9, and L11 HA insertion mutants. These mutants remained capable of transferrin binding and use of transferrin as a sole iron source, as long as $\mathrm{TbpB}$ was co-expressed. If $t b p B$ was also mutated, the double mutants were rendered completely incapable of transferrin-iron internalization. The phenotypes of these mutants suggest that L2, L9, and L11 represent regions of TbpA that are important for iron removal from transferrin, without which TbpA is unable to function in the absence of TbpB.

TbpB shares similarity with other surface-exposed lipoproteins, but until recently none had been crystallized. From sequence analysis, it was clear that the gonococcal TbpB was comprised of two domains that shared limited sequence similarity, reminiscent of the ligand to which it binds, transferrin. We demonstrated that the amino-terminal half of gonococcal $\mathrm{TbpB}$ retained the ability to bind human transferrin following SDS-PAGE and transfer to solid support (Cornelissen et al., 1997a). Truncation of this binding domain from either end abolished binding. We further demonstrated that the amino-terminal half of $\mathrm{TbpB}$ contained most of the sequence diversity when five gonococcal sequences were compared. There were, however, six regions of sequence conservation that are probably important for export to the cell surface or for transferrin binding.

We employed the same approach to mapping gonococcal TbpB membrane topology as was described above for TbpA. We expressed TbpB-HA fusion proteins in the gonococcus, each of which contained an HA epitope at a distinct position (DeRocco and Cornelissen, 2007). All of the HA epitopes were accessible on the gonococcal cell surface, consistent with complete surface exposure of TbpB beyond the lipid modified, mature amino-terminal cysteine residue. There were only four epitope insertions that decreased transferrin binding by TbpB. Three insertion points were within the amino-terminal lobe that is responsible for efficient transferrin binding following SDS-PAGE and electroblotting to solid support. The fourth insertion point was within the C-terminal half of the protein, disruption of which resulted in decreased transferrin binding to the whole cell surface. When mutations in the aminoand carboxy-terminal halves were combined in the same strain, the resulting mutant $\mathrm{TbpB}$ was completely devoid of transferrin binding capabilities. These results suggest that the amino- and carboxy-terminal lobes of TbpB share both sequence and functional redundancy. Elimination of binding to one lobe only decreased transferrin interaction with the cell surface, while elimination of both domains abrogated all binding. Recently, the crystal structure for the smaller but related TbpB from Actinobacillus pleuropneumoniae was described (Moraes et al., 2009). The gonococcal protein is expected to assume a similar conformation, which is reminiscent of other lipid-modified neisserial proteins, including the factor $\mathrm{H}$ binding protein (Schneider et al., 2009). The A. pleuropneumoniae TbpB has two lobes, each of which has two distinct domains: a small beta-barrel and a curved, $\beta$-strand rich region denoted as a "handle" (Moraes et al., 2009). The amino- and carboxy-terminal domains of gonococcal TbpB are expected to resemble those of A. pleuropneumoniae TbpB and are depicted in Figure $\mathbf{1}$ as trapezoid shapes.
Both lobes of gonococcal TbpB retain transferrin binding domains (DeRocco and Cornelissen, 2007) and thus both are expected to participate in the process of iron acquisition from transferrin.

The gonococcal Tbp proteins are expected to form a complex, particularly in the presence of transferrin. We have detected TbpB in pull-down assays with TbpA and TbpA-specific antisera (Kenney, 2002), suggesting that even in the absence of transferrin the two proteins associate with each other. Surface exposure characteristics and binding kinetics differ depending upon whether the Tbp proteins are expressed independently or together, again suggesting complex formation at the cell surface (Cornelissen and Sparling, 1996; Cornelissen et al., 1997b). The transferrin binding affinities of both proteins for human transferrin are in the nanomolar range; however, $\mathrm{TbpB}$ specifically associates with the ferrated form of transferrin whereas TbpA interacts with both ferrated and apo forms of the glycoprotein (Cornelissen and Sparling, 1996). This observation led us to test the association and dissociation rates for the individual proteins. We found that both association and dissociation was facilitated by the presence of $\mathrm{TbpB}$, suggesting that the ability of $\mathrm{TbpB}$ to discriminate between ferrated and apo-transferrin enhances both association and dissociation kinetics (DeRocco et al., 2008). Employing an in vitro technique and gonococcal membrane fragments, we determined that both TbpA and TbpB have the ability to remove iron from human transferrin (Siburt et al., 2009). In this in vitro system, the ferric binding protein, FbpA, was freely available in solution and served as the ferric iron acceptor molecule. Cumulatively, our studies suggest that while both $\mathrm{TbpA}$ and $\mathrm{TbpB}$ have the capacity to strip ferric iron from transferrin at the cell surface, in an intact cell, only TbpA has the capacity to transport iron across the outer membrane and into the periplasm.

While the precise mechanism of iron removal from transferrin and subsequent transport into the periplasm is not completely understood, our current model (Figure 3) involves both TbpA

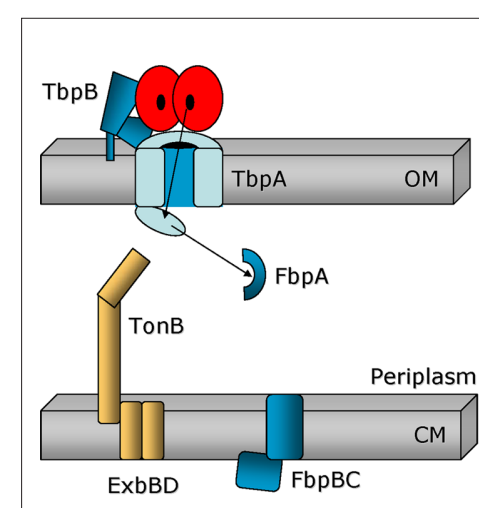

1. Fe-Tf binds to $\mathrm{TbpA}+\mathrm{TbpB}$

2. TbpA and TbpB participate in iron extraction from $\mathrm{Fe}$-Tf

3. Iron interacts with plug domain for transport

4. TonB initiates transport by pulling the plug

5. Localized denaturation of the plug results in iron release

6. Iron is captured by $\mathrm{FbpA}$

7. TbpB facilitates apo-Tf release from surface

FIGURE 3 | Model of the mechanism of iron transport from transferrin through the gonococcal outer membrane. TbpA is shown as a bisected barrel with an amino-terminal plug domain exposed in the periplasm. TbpB is shown as a bi-lobed protein protruding from the gonococcal cell surface, tethered to the outer membrane by an amino-terminal lipid moiety. Human transferrin is shown as a bi-lobed structure, each lobe of which is capable of binding an atom of iron. Seven putative steps in iron acquisition from transferrin are listed on the right. The arrows depict the direction of iron import and the hypothetical, transient interaction between iron and the plug domain. 
and $\mathrm{TbpB}$ playing roles in iron removal from transferrin at the cell surface (Siburt et al., 2009). After iron is extracted, we propose that the ferric ion interacts with the plug domain of TbpA. The HA epitope insertion in the plug domain resulted in a mutant that bound transferrin with lower affinity but was incapable of iron internalization (Yost-Daljev and Cornelissen, 2004). This observation prompted us to create a series of site-specific mutations in conserved, charged residues of the plug domain near the point of insertion of the HA epitope (Noto and Cornelissen, 2008). A triple mutant in which a conserved EYE motif was replaced by three alanine residues was generated. This mutant strain was incapable of transferrin-iron utilization unless TbpB was co-expressed. This phenotype is consistent with a role for this region in iron extraction and/or iron binding by the plug domain. The model of transport of iron through TonB-dependent transporters, developed by study of the vitamin $B_{12}$ transporter of E. coli (Gumbart et al., 2007) suggests that plug unfolding upon interaction with TonB (see below) results in presentation of substrate at the periplasmic face of the beta-barrel. We hypothesize (Figure 3) that a similar mechanism would result in transport of iron, bound by the plug, through the barrel and allow the presentation of iron at the periplasmic face of TbpA.

\section{PERIPLASMIC BINDING PROTEIN-DEPENDENT, ABC TRANSPORT OF FERRIC IRON FROM TRANSFERRIN}

After ferric iron has been transported through the TbpA betabarrel, the ferric binding protein, FbpA, sequesters the ion in the periplasm (Chen et al., 1993). FbpA has been called a "bacterial transferrin" because binding by this $37 \mathrm{kDa}$ protein to iron resembles that of a single lobe of transferrin in terms of coordinating residues and the need for a synergistic anion (Nowalk et al., 1994; Taboy et al., 2001; Dhungana et al., 2003). Using a modified H/D exchange approach, we demonstrated that apo-FbpA binds directly and specifically to TbpA (Siburt et al., 2009). This interaction would allow for an efficient hand-off from the TbpA plug domain to the associated apo-FbpA. After FbpA becomes ferrated, its affinity for TbpA is decreased, facilitating liberation of the holo-FbpA and passage of the sequestered iron through the periplasmic space. Holo-FbpA then associates with the cytoplasmic permease protein, $\mathrm{FbpB}$, which accomplishes transport from the periplasm into the cytoplasm employing the FbpC protein and ATP hydrolysis for energization. One publication reported that iron transport into the gonococcus was accomplished in the absence of a functional FbpC protein (Sebastian and Genco, 1999). This observation suggests that other ATP binding proteins, of which there are many predicted in the gonococcal genomes, might be able to replace FbpC in this energization step.

\section{LACTOFERRIN-IRON ACQUISITION}

As shown in Figure 1, the lactoferrin-iron acquisition system, comprised of $\mathrm{LbpA}$ and $\mathrm{LbpB}$, resembles the transferrin iron acquisition system. LbpA is a TonB-dependent transporter (Biswas and Sparling, 1995); LbpB is a lipoprotein (Biswas et al., 1999). Gonococcal LbpA from strain FA19 is $46 \%$ identical and $65 \%$ similar to gonococcal TbpA from the same strain (Biswas and Sparling, 1995). LbpB is 31\% identical to TbpB; however LbpB contains two unique stretches of anionic residues, which are absent from TbpB (Biswas et al., 1999). As is the case for the Tbps, the Lbps are encoded by contiguous genes with the $l b p B$ gene located upstream of the $l b p A$ gene. In contrast to the $t b p$ operon, the $l b p B$ and $l b p A$ genes actually overlap slightly and are not separated by a region of potential secondary structure. About half of gonococcal isolates have lost the capacity to express the Lbps due to the presence of a large deletion that removes the entire $l b p B$ gene and the $5^{\prime}$ terminus of the $l b p A$ gene (Anderson et al., $2003)$. In addition, the $l b p B$ gene, if present, is subject to phase variation due to a poly $\mathrm{C}$-tract within the coding region. While LbpA is required for utilization of human lactoferrin as a sole iron source, LbpB is not (Biswas et al., 1999). Variants in which $\mathrm{LbpB}$ is not expressed due to phase variation, retain the ability to express LbpA, and the ability to grow on lactoferrin. LbpB does not apparently bind lactoferrin independent of LbpA (Biswas et al., 1999), in contrast to the situation with the Tbps, which both retain the ability to bind specifically to human transferrin. Detailed structure-function analyses have not been conducted to date with the gonococcal Lbps. Thus the mechanism of lactoferrin binding, iron extraction, TonB energization and iron transport into the periplasm is unclear. The FbpABC system is known to receive and transport lactoferrin-bound iron (Chen et al., 1993) through the periplasm and across the cytoplasmic membrane as shown in Figure 1.

\section{HEMOGLOBIN-IRON ACQUISITION}

Neisseria gonorrhoeae is capable of utilizing both free heme and heme bound to hemoglobin to fulfill its iron requirement. No specific receptor has been defined for free heme; however, hemoglobin is employed by virtue of expression of a third two-component iron acquisition system (Chen et al., 1996, 1998). As shown in Figure 1, the topology of the hemoglobin-iron acquisition system, comprised of $\mathrm{HpuA}$ and $\mathrm{HpuB}$, is expected to resemble those employed for transferrin-and lactoferrin-iron utilization. HpuB is encoded downstream of HpuA; the $h p u B$ gene encodes the TonB-dependent transporter whereas the $h p u A$ gene encodes the lipidated component of the system. The HpuB protein at $\sim 90 \mathrm{kDa}$ (Chen et al., $1996)$ is somewhat smaller than either TbpA or LbpA $(\sim 100 \mathrm{kDa})$, but is similar in size to most other TonB-dependent transporters (Table 1). Similarly, the lipoprotein component, HpuA, is approximately half the size of either TbpB or LbpB (Chen et al., 1998). Given the recent insights into the structure of $\mathrm{TbpB}$ (Moraes et al., 2009), one could speculate that HpuA is composed of a single lobe with only one beta-barrel and a single "handle" domain (as shown in Figure 1). Interestingly, and in contrast to the transferrin- and lactoferrin-iron acquisition systems, hemoglobin binding requires the expression of both $\mathrm{HpuA}$ and $\mathrm{HpuB}$, consistent with the hypothesis that the two proteins form a heteromultimer in order to constitute the obligate hemoglobin binding pocket (Chen et al., 1998). Also consistent with the single binding pocket hypothesis is the observation that hemoglobin-iron utilization is dependent upon expression of both HpuA and $\mathrm{HpuB}$, unlike the TbpAB system which only requires expression of TbpA. Also in stark contrast to the transferrin and lactoferrin binding proteins, HpuA and B do not exclusively interact with human hemoglobin as these gonococcal proteins recognize the hemoglobins from non-human sources as well. Point mutations in HpuB allow 
Table 1 | TonB-dependent transporters encoded in the gonococcal genome.

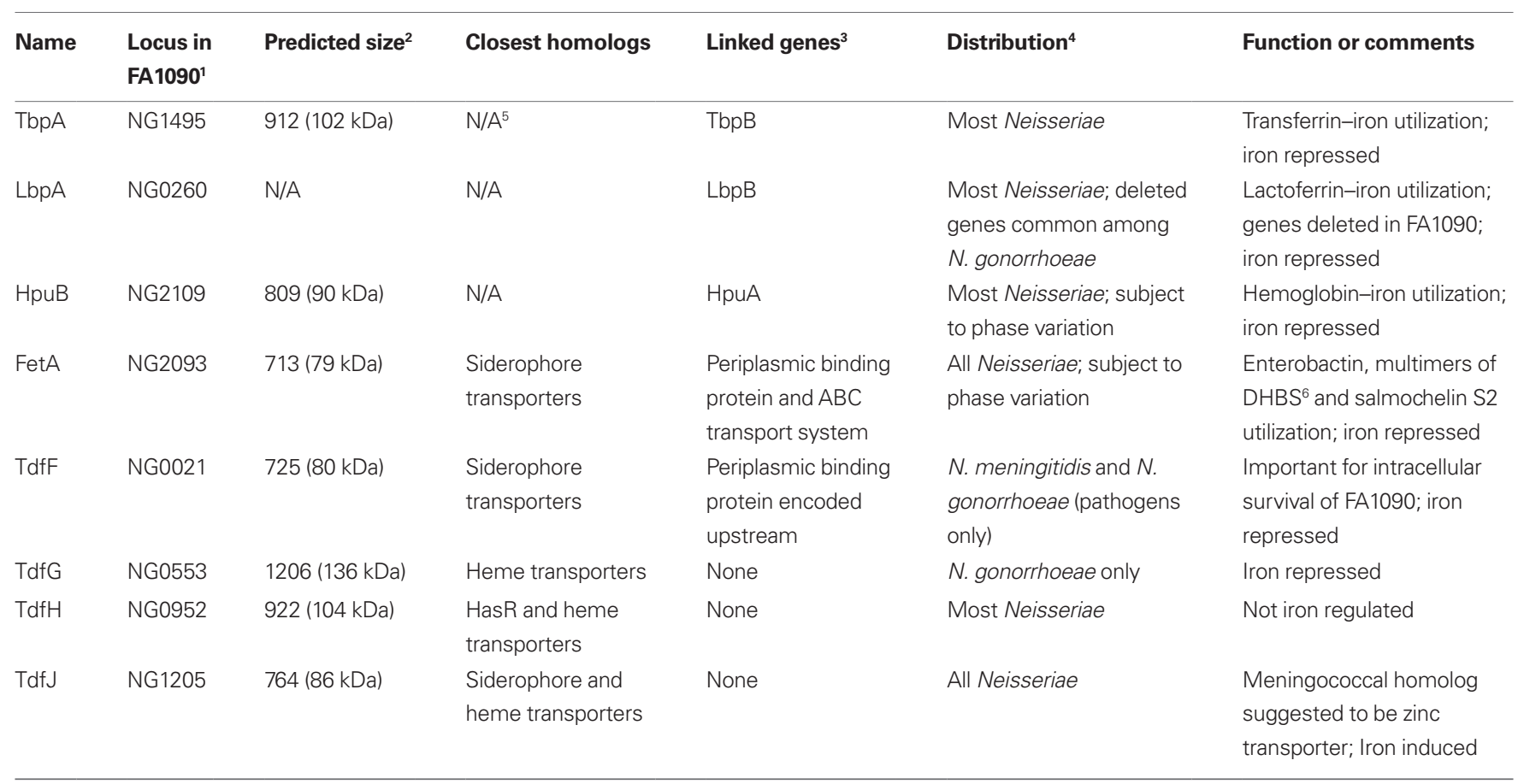

'Locus corresponds to the gene ID in the following database: stdgen.northwestern.edu. Genome sequence of FA1090 was determined at the University of Oklahoma. ${ }^{2}$ Predicted size in number of amino acids followed by predicted molecular weight in parentheses. ${ }^{3}$ Linked genes that have homologs known to be involved in iron

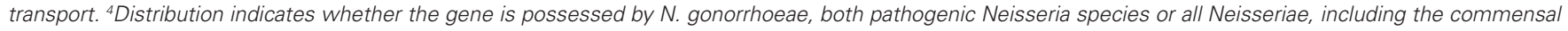
species. ${ }^{5} \mathrm{~N} / \mathrm{A}$, not applicable. ${ }^{6}$ Dihydroxybenzoylserine.

hemoglobin utilization in the absence of the lipoprotein partner protein, HpuA (Chen et al., 2002). The $\Delta h p u A, h p u B$ point mutants grew slightly better than the $\Delta h p u A, h p u B+$ parent in the presence of limiting amounts of heme. The point mutations mapped to the beta-barrel portion of the TonB-dependent transporter, suggesting that transmembrane passage of the heme was enhanced in the mutants. Finally, in the absence of HpuA, growth by the hemoglobin+ point mutants was abrogated in the presence of albumin, which binds and makes inaccessible heme outside the cell. The authors therefore concluded that an important function for HpuA is to retain heme in close proximity to the cell to make transport through the TonB-dependent transporter more efficient (Chen et al., 2002). Beyond these studies, detailed structure-function analyses have not been conducted on the gonococcal $\mathrm{HpuAB}$ proteins. Subsequent to transport through $\mathrm{HpuB}$, heme is presumably bound by a periplasmic binding protein (PBP) for transit to the cytoplasmic membrane. Likewise, a heme-specific cytoplasmic membrane permease is expected to be necessary for entry into the cytoplasm. However, no such PBP-dependent, ABC transport system has yet been described.

A second TonB-dependent hemoglobin-iron transporter is expressed by the closely related $N$. meningitidis (Stojiljkovic et al., 1996). HmbR is similar to HpuB, but in contrast to the systems described above, meningococcal $\mathrm{HmbR}$ is not encoded proximate to a companion lipoprotein. Moreover, none of the gonococcal strains sequenced to date have the capacity to express $\mathrm{HmbR}$ as the loci that would encode this protein are pseudogenes.

\section{SINGLE COMPONENT SYSTEMS FOR IRON TRANSPORT FetA}

The fetA gene was formerly called $\operatorname{frpB}$ (Beucher and Sparling, 1995) and encodes a TonB-dependent transporter of approximately $80 \mathrm{kDa}$. The ferric enterobactin transporter (FetA) protein was renamed when Carson et al. (1999) demonstrated that binding and acquisition of ferric-enterobactin was enhanced when FetA was expressed. Enterobactin is a catecholate-type siderophore that is produced by a variety of bacteria, including E. coli (O'Brien and Gibson, 1970), Salmonella species (Pollack and Neilands, 1970), and Klebsiella species (Perry et al., 1979). The gonococcal fetA gene lies immediately upstream of a cluster of genes that putatively encode components of a PBP-dependent, ABC transport system. Carson et al. (1999) demonstrated that $f e t B$ mutants were defective in enterobactin utilization. The gene products encoded downstream of FetA are shown in Figure 4 and are labeled as FetB, the PBP, and FetCDEF, putative members of the $\mathrm{ABC}$ transport system. There is one ORF (NG2089) within the cluster that has no homologs but is conserved among the Neisseria species; therefore, this protein's role in iron uptake is not obvious. This ORF corresponds to the FetE protein shown in Figure 4. While FetA was known to transport ferric-enterobactin (Carson et al., 1999), we recently demonstrated (Hollander et al., in revision) that this transporter is also critical for import of other catecholate siderophores including dimers and trimers of dihydroxybenzoylserine (DHBS) and salmochelin S2 (the linearized form of salmochelin). Salmochelin is a diglucosylated form of enterobactin that is made by some pathogens, 


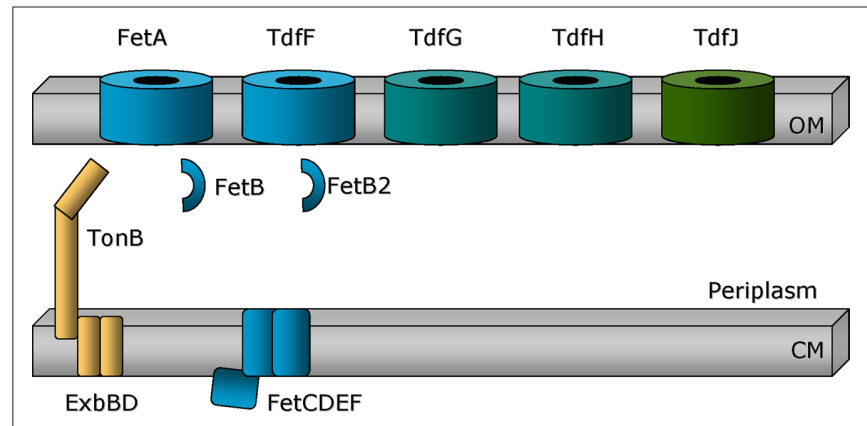

FIGURE 4 | Single component iron transport systems. Fet $A$ and the uncharacterized TonB-dependent transporters (TdfF, TdfG, TdfH, and TdfJ) are shown as barrels traversing the outer membrane. TonB, ExbB, and ExbD (gold) are depicted as attached to or imbedded within the cytoplasmic membrane (CM). The periplasmic binding protein FetB is encoded downstream of FetA and is expected to transport ferric-siderophores across the periplasm to a cytoplasmic membrane permease. Genes located immediately downstream of that encoding FetB are hypothesized to fulfill this function. Encoded near TdfF is a putative periplasmic binding protein annotated as FetB2, reflecting its sequence similarity with FetB.

including uropathogenic E. coli (Hantke et al., 2003). Thus, the FetA transporter appears to have broad specificity for catecholate-type xenosiderophores. FetA expression is iron repressed and also subject to phase variation. A poly C-tract within the promoter region preceding the fet $A$ gene results in modulated expression levels from very high to very low (Carson et al., 2000).

\section{TdfF}

When the first gonococcal genome sequence was being completed, Turner et al. (2001) identified three uncharacterized TonBdependent transporters by their similarity to other proteins in this family. These putative transporters were named TonB-dependent function (Tdf) F, G, and H (Figure 4). As shown in Table 1, these transport proteins were similar to siderophore and heme transporters from other Gram-negative bacteria. With subsequent sequence analysis, we identified a fourth putative transporter, which we named TdfJ (Table 1; Hagen and Cornelissen, 2006).

To discern whether any of the TonB-dependent transporters were important for gonococcal survival within human epithelial cells, we modified an assay developed by Larson et al. (2002) to evaluate intracellular survival of $N$. meningitidis. We first tested whether a tonB mutant of gonococcal strain FA1090 was inhibited for growth within human cervical epithelial cells. We found that the tonB mutant was indeed impaired for survival and that the addition of iron rescued the mutant (Hagen and Cornelissen, 2006), suggesting that the defect was related to an iron acquisition defect in the ton $B$ mutant. We then tested both characterized TonB-dependent transporters (TbpA and FetA) and the uncharacterized TonBdependent transporters ( $\mathrm{TdfF}, \mathrm{G}, \mathrm{H}$, and $\mathrm{J}$ ) for their participation in this survival phenotype. The only mutant that was defective for intracellular survival in these assays was the $t d f F$ mutant (Hagen and Cornelissen, 2006). Like the tonB mutant, addition of excess iron overcame the survival defect, suggesting that the TdfF transporter participated in intracellular iron acquisition and that excess iron could bypass the need for high affinity transport through TdfF.
Upstream of the $t d f F$ gene is a gene that putatively encodes a PBP related to iron transport. This gene is annotated in the FA1090 genome database as $\mathrm{fet} B 2$ due to its similarity to the protein encoded within the fet gene cluster. TdfF expression is iron regulated, but is only detected when gonococci are grown in cell culture medium plus serum (Hagen and Cornelissen, 2006). This is consistent with the inability of Turner et al. (2001) to detect TdfF expression in gonococcal growth medium, even under iron stressed conditions. Likewise, in microarray analyses of the iron-dependent gonococcal transcriptome, $t d f F$ expression has never been reported (Ducey et al., 2005; Jackson et al., 2010). We hypothesize that the putative AraC-like transcriptional regulator, $\mathrm{MpeR}$, encoded in close proximity to $t d f F$ may activate expression of TdfF in the presence of the appropriate inducing stimulus. The molecular identity of this stimulating molecule(s) is currently under investigation. The entire locus between $t d f F$ and mpeR is unique to the pathogenic Neisseria species as it is completely absent from commensal Neisseriae (Snyder and Saunders, 2006; Marri et al., 2010). This observation suggests that iron acquisition via the TdfF-dependent pathway and optimized MpeR-dependent gene regulation are important virulence factors, distinguishing the pathogens from the commensals.

\section{OTHER UNCHARACTERIZED TONB-DEPENDENT TRANSPORTERS}

TdfG is an extraordinarily large TonB-dependent transporter at $\sim 136 \mathrm{kDa}$ and is encoded by all gonococcal genomes; however, protein expression was only detected by Turner et al. in $17 \%$ of gonococcal strains (Turner et al., 2001). TdfG is most similar to heme transporters and was shown by Turner et al. (2001) to be iron repressed. A knock-out mutant of strain FA1090 that was unable to express TdfG retained the ability to utilize heme as a sole iron source. All gonococcal genomes sequenced to date have an intact $t d f G$ gene; however the sequences fall into two distinct clades, showing only $48-49 \%$ sequence similarity to each other (data not shown). The biological significance of these different $t d f G$ gene families among different gonococcal strains is unclear but may have contributed to the inability to detect protein expression in the majority of gonococci using an antibody probe despite the presence of full-length genes. TdfH is also a large TonB-dependent transporter $(\sim 104 \mathrm{kDa})$ with similarity to heme transport systems. $\mathrm{TdfH}$ mutants similarly retained the ability to grow on free heme as a sole iron source (Turner et al., 2001). Moreover, expression of recombinant $\mathrm{TdfH}$ in a hemA mutant of $E$. coli was not sufficient to allow heme utilization, even when the neisserial TonB, ExbB and ExbD proteins were co-expressed. Cumulatively, these data suggest that neither TdfG nor TdfH is a TonB-dependent transporter for free heme (Turner et al., 2001); however, the genes were not simultaneously inactivated in the gonococcus. If the functions of TdfG and TdfH are redundant with respect to iron acquisition, their phenotypes may only be obvious in a mutant lacking both transporters.

TdfJ is an $86 \mathrm{kDa}$ TonB-dependent transporter that shares similarity with siderophore and heme transporters. The $t d f$ gene is present in all Neisseriae, including pathogens and commensals. Microarray studies (Ducey et al., 2005; Jackson et al., 2010) and our unpublished, confirmatory RT-PCR experiments demonstrate that $t$ dfJ expression is iron induced. A recent publication indicates that the meningococcal TdfJ homolog (NMB0964) facilitates zinc 
acquisition and thus the authors renamed the meningococcal protein $\mathrm{ZnuD}$ (Stork et al., 2011). ZnuD is regulated by the putative zinc-dependent regulator, Zur, but expression of $\mathrm{ZnuD}$ in the meningococcus was not subject to iron regulation. Similar studies have not been reported for gonococcal TdfJ, so the function of this protein and its contribution to growth of the gonococcus is not currently known. However, the mechanisms that regulate expression of these TonB-dependent transporters in the two pathogenic Neisseriae appear to be distinct.

\section{TonB and iron transport across the gonococcal outer membrane}

While the mechanism by which TonB harnesses energy and transduces it to energize the outer membrane iron transporters has not been completely defined in any system, it is well established that TonB, in complex with ExbB and ExbD is charged by the proton motive force generated at the cytoplasmic membrane (Postle and Larsen, 2007). TonB, in its energized state, physically interacts with TonB-dependent transporters. One conserved domain within the plug domain of the transporters has been called the "TonB-box" (Cadieux et al., 2000). Defined domains of TonB have been demonstrated to interact directly with the TonB-box (Pawelek et al., 2006) and in so doing, facilitate substrate transport across the outer membrane. We generated a TonB-box (Figure 2) mutant of gonococcal TbpA and showed that while transferrin binding was unaffected, transferrin-iron acquisition was completely prevented (Cornelissen et al., 1997b). The TonB-box mutant presented TbpB at the cell surface in a distinct conformation, resulting in a new protease sensitivity pattern. This observation allowed us to conclude that the energization state of TbpA resulted in a conformational change in $\mathrm{TbpB}$, consistent with a physical interaction between the two outer membrane proteins. Additionally, the TonB-box mutant was incapable of transferrin release from TbpA, suggesting that the energy-dependent step in transferrin-iron transport is necessary for ligand release to occur. TonB directly interacted with TbpA, but the association was inhibited when the TonB box of TbpA was mutated (Kenney and Cornelissen, 2002). The ability of TonB to copurify with TbpA did not depend upon the presence of the natural ligand, transferrin (Kenney and Cornelissen, 2002), prompting us to conclude that the natural signal triggering TonB interaction with TbpA was in fact iron binding by the plug domain.

Most current models of TonB function are based upon the known crystal structures of TonB-dependent transporters and TonB. These models suggest that the most efficient means to accomplish vectorial ligand transport through the beta-barrel is by TonB first binding to the TonB-box and then initiating a pulling force on the plug domain (Shultis et al., 2006). This would result in a localized denaturation or unfolding of the plug, allowing movement of substrate through the barrel and presentation at the periplasmic face of the transporter. With regard to gonococcal transferrin-iron acquisition, we propose (Figure 3 ) that both TbpA and TbpB participate in iron removal at the cell surface. The plug domain of TbpA may be at least partially surface accessible and therefore may also participate in iron removal from transferrin. We postulate that the plug domain interacts with the extracted ferric iron and that the EYE motif in some way participates in this process. We further propose that TonB then interacts with the TonB-box of TbpA, a pulling force is exerted resulting in plug unraveling, at which point the ferric ion is presented to apo-FbpA, which we demonstrated interacts with TbpA. Apo-transferrin release would re-set the system to baseline but would not occur if all of the previous steps had not been accomplished, as in the TonB-box mutant.

\section{TonB-INDEPENDENT USE OF IRON AND XENOSIDEROPHORES}

While use of ferric siderophores in other Gram-negative bacteria is accomplished via TonB and TonB-dependent transporters, several iron sources are employed by N. gonorrhoeae in a Ton-independent manner. Ferric citrate, free heme (Biswas et al., 1997), and some xenosiderophores (Strange et al., 2011) are acquired by the gonococcus in pathways that do not depend upon expression of TonB or any of the individual TonB-dependent transporters. The mechanisms by which ferric citrate and heme are internalized have not been explored, but we recently demonstrated that gonococcal strain FA19 utilizes the xenosiderophores ferric enterobactin, DHBS, and salmochelin S2 in a pathway that is TonB-independent (Strange et al., 2011). Use of the xenosiderophores by strain FA19 is limited to an inefficient, Ton-independent system, which requires expression of the FbpABC proteins (Figure 5). When the Fbp proteins were not expressed, xenosiderophore utilization was completely prevented. Since mutants lacking the outer membrane protein complexes Mtr or PilQ remained competent for Ton-independent iron acquisition (Strange et al., 2011), we suggest that the xenosiderophores permeate the outer membrane passively, perhaps through porins (Figure 5). This is in contrast to utilization of enterobactin, DHBS, and S2 by gonococcal strain FA1090, which occurs in a TonB- and FetAdependent pathway (Figures 4 and 5; Carson et al., 1999; Hollander

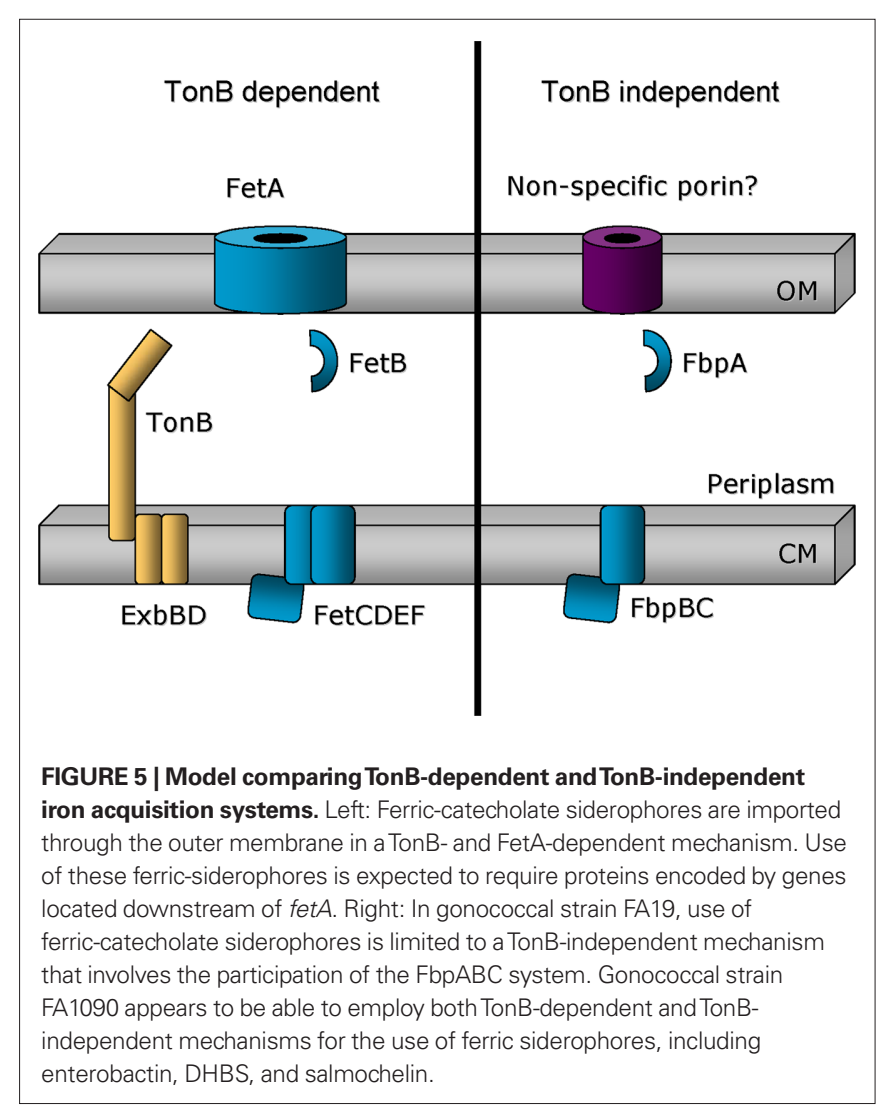


et al., in revision). We propose that all gonococcal strains have the capacity to internalize iron from xenosiderophores by both FetAdependent and FetA-independent pathways. In FA1090, xenosiderophore-dependent growth by the ton $B$ and $f e t A$ mutants was diminished relative to wild-type, but not eliminated, suggesting that both TonB-dependent and TonB-independent pathways are functional. FA19 however is limited to the TonB-independent pathway. Why might FA19 be incapable of Fet-mediated internalization of enterobactin and its derivatives? One reason could be because FetA expression varies between strains and isolates due to the poly C-tract in the promoter region (Carson et al., 2000). Another possible explanation could be related to the observation that the genome sequence of gonococcal strain FA19 contains a frame-shift mutation within $n g 2090$ (encoding FetD in Figure 5). We have confirmed by direct sequencing of a PCR product amplified from FA19 chromosomal DNA that this lesion represents a genuine mutation and is not a sequencing error (data not shown). This mutation would result in a truncated FetD protein and may prevent expression of downstream genes as well. We hypothesize that one or both of these genetic differences between strains FA1090 and FA19 result in a defective Fet system and reliance on the TonB-independent uptake pathway for uptake of iron from xenosiderophores in strain FA19.

We have also detected strain-specific, Ton-independent growth phenotypes with respect to intracellular survival. While we demonstrated that intracellular survival of strain FA1090 was TonB- and TdfF-dependent (Hagen and Cornelissen, 2006), we found that this was not the case in two other gonococcal strains. Intracellular survival of strains FA19 and MS11 did not depend upon expression of either TonB or TdfF (Zola et al., 2010). This suggested that something unique to these strains allowed a bypass of the need for high affinity iron acquisition through the TonB system and TonBdependent transporters. Both FA19 and MS11 differ from FA1090 by possessing the gonococcal genetic island (GGI), which encodes a type IV secretion system (Hamilton et al., 2005). In collaboration with Joe Dillard's group, we created double mutants lacking both the Ton system (a polar mutation in $\operatorname{ton} B$ ) and the GGI. This double mutant was incapable of intracellular survival whereas the single mutants lacking either the Ton system or the GGI remained competent to survive within cervical epithelial cells (Zola et al., 2010). We mapped the key region of the GGI to the structural genes that encode critical components of the type IV secretion apparatus. None of the accessory proteins or known secreted proteins were involved in the TonB bypass mechanism. We proposed three possible explanations for these observations. First, the type IV secretion apparatus may secrete a reductase allowing the gonococcus to acquire ferrous iron in a TonB-independent pathway. Second, the type IV secretion apparatus may simply form an indiscriminate pore through the cell envelope, allowing ferric iron entry. And finally, the type IV secretion system may facilitate the entry of the gonococcus into an intracellular niche that does not require high affinity iron acquisition via the Ton system.

\section{CONTROLLED EXPRESSION OF IRON ACQUISITION SYSTEMS REPRESSION}

All of the characterized iron acquisition systems are subject to irondependent repression. The most common mechanism employed for iron-dependent repression is mediated through the function of the ferric uptake regulator (Fur) protein. Iron-regulated expression of the transferrin and lactoferrin binding proteins is known to be Fur dependent (Thomas and Sparling, 1996). As is the case in other Gram-negative bacteria (Escolar et al., 1999) when Fur is complexed with ferrous iron, the protein dimerizes and binds to a DNA sequence called a "Fur box," which generally overlaps with the promoter region preceding iron-repressed genes (Sebastian et al., 2002; Jackson et al., 2010). However, there are examples of Fur binding sites within structural genes or overlapping transcriptional start sites. In any event, binding of Fur results in the inaccessibility of the promoter to the RNA polymerase or the inability of the RNA polymerase to move processively down the template strand to transcribe the entire gene. When iron is limiting, the apo-Fur protein no longer functions as a repressor, thus gene expression is enabled. This simple mechanism results in expression of iron acquisition systems only under conditions of iron depletion.

\section{ACTIVATION}

There are no characterized activators of gonococcal iron transport systems. However, we recently discovered that an iron-repressed, AraC-like regulator activates expression of $f e t A$ (Hollander et al., in revision). MpeR was previously shown to regulate the expression of $m t r F$, which in turn controls the activity of the MtrCDE efflux pump (Folster and Shafer, 2005). We subsequently observed that MpeR is encoded upstream of TdfF and an iron-related PBP annotated as FetB2 (see above). When mpeR was inactivated, we were surprised to discover that another TonB-dependent transporter, FetA, was no longer expressed. We determined that $\mathrm{MpeR}$ binds to the region upstream of the fetA gene, consistent with a direct mechanism of activation. These observations cumulatively link expression of antimicrobial efflux systems with iron transport systems through the iron-regulated expression of a key regulator, MpeR. As noted above, the mpeR - tdff locus is unique to the pathogenic Neisseria species, which suggests that optimum expression of efflux systems and iron transport systems represents an important virulence mechanism.

\section{TRANSLATIONAL IMPLICATIONS HUMAN AND ANIMAL INFECTION EXPERIMENTS}

The transferrin-iron acquisition system was the first to be tested for its influence on the outcome of experimental human infections. Gonococcal strain FA1090 was chosen for human infection experiments because the Opa protein profile has been well characterized in this background (Connell et al., 1990; Jerse et al., 1994). FA1090 is also naturally unable to utilize lactoferrin as a sole iron source due to the inability to express either of the lactoferrin binding proteins. We created a mutant derivative of gonococcal strain FA1090 that no longer expressed either of the Tbps, effectively generating a double mutant lacking the ability to employ either transferrin or lactoferrin as sole iron sources. This mutant was completely unable to initiate urethritis in a human male infection model, whereas infection with the wild-type FA1090 resulted in urethritis within 3-4 days of inoculation (Cornelissen et al., 1998). These results indicated that expression of the Tbps was critical for the first steps in initiating infection on a human male urethra. While suggestive, these findings do not definitively implicate the use of transferrin as a key for initiating infection since the Tbps could conceivably serve multiple functions in vivo, as has been documented with other gonococcal 
proteins. Subsequently, Anderson et al. (2003) generated a derivative of FA1090 in which the lactoferrin binding proteins were expressed, but the genes encoding the Tbps were deleted. Unlike the $t b p$ mutant parent, the $\mathrm{Lbp}^{+}$variant was capable of causing urethritis in human male volunteers. These results suggested that expression of the Lbp proteins, while not universal among gonococci, was sufficient to restore infectivity. The reason that so many gonococci have lost the capacity to express the lactoferrin-iron acquisition system, in spite of its obvious in vivo utility, remains unclear.

The hemoglobin-iron acquisition system, comprised of HpuA and $\mathrm{B}$ described above, is subject to phase variation due to a poly $\mathrm{G}$ tract within the HpuA coding region. Depending upon the number of $\mathrm{G}$ residues, the HpuA gene is either in- or out-of-frame. Anderson et al. (2001) demonstrated that variants capable of expressing HpuA were selected for in vivo, but only under specific conditions. By screening gonococcal strains isolated directly from infected men and women, these investigators determined that women in the early phase of their menstrual cycle disproportionately harbored gonococcal isolates that expressed HpuA and grew on hemoglobin. These results suggest that the ability to grow on hemoglobin is selected for by the intermittent presence of this protein in the human genital tract.

Jerse and colleagues (Jerse, 1999) have developed a mouse model of lower female genital tract colonization to facilitate studies of gonococcal pathogenesis and vaccine development. While the gonococcus is clearly species restricted to humans for normal genital tract infection, the mouse model mimics lower genital tract infection in humans in many ways. We tested gonococcal mutants of strain FA1090 individually lacking the ability to employ transferrin, lactoferrin, hemoglobin or ferric enterobactin for their ability to colonize the genital tract of female mice (Jerse et al., 2002). All mutants were competent for colonization, including a gonococcal tonB mutant (data not shown). These results imply that other sources of iron are available in the female genital tract, and that gonococcal access to this nutrient in the mouse does not depend upon expression of TonB-dependent systems or transporters. Perhaps due to the lower $\mathrm{pH}$ of the female genital tract and the co-localization of lactobacilli, iron may be more accessible, and thereby may not require high affinity acquisition systems for entry.

\section{VACCINE DEVELOPMENT}

As described above, many of the characterized TonB-dependent transport systems are subject to high frequency phase variation or are not universally expressed by gonococci. The exception to this observation is the transferrin-iron acquisition system, which is ubiquitously expressed by gonococci. The Tbps are obviously expressed in humans during infection; if this were not the case, the tbp mutant would have been without a phenotype in the human infection model. We detected low but measurable antibody titers in the serum and vaginal wash specimens from naturally infected women and men (Price et al., 2004). These antibody levels were well below those associated with a protective response, as was detected against tetanus toxoid. While the Tbps are clearly expressed in vivo (Agarwal et al., 2005), the mere presence of these proteins within the context of a natural infection is insufficient to initiate a high-titer, protective immune response (Price et al., 2004), consistent with the lack of immunity following natural infections.
A gonococcal vaccine has been sought for many years, but due to high frequency phase and antigenic variation and immune evasion and subversion strategies, no vaccine formulation of protein antigens or surface structures has met with any success. Because the gonococcal Tbps are ubiquitously expressed, not subject to high frequency variation and necessary to initiate infection in human males, we have focused vaccine development efforts on these proteins. When conjugated to the B subunit of cholera toxin, both TbpA and TbpB generated IgG in the serum of intranasally vaccinated mice (Price et al., 2005). TbpB-specific antibody levels were significantly higher than those generated against TbpA. Antibodies elicited against the Tbp proteins were bactericidal in the presence of human complement. Mucosal antibodies, detected in the genital tract, were also generated against the Tbp proteins. We have also immunized mice with portions of the Tbp proteins, genetically fused to the A2 domain of cholera toxin (Price et al., 2007). Co-expression of the fusion proteins with the B subunit of cholera toxin resulted in a complex comprised of the $\mathrm{B}$ pentamer and immunogenic regions of the Tbps. We chose the amino-terminal lobe of TbpB and surface-exposed loop 2 of TbpA for this analysis. Antibodies generated following intranasal vaccination with these epitope-specific antigens were detected in the serum and mucosal secretions. Serum antibodies in the presence of human complement were bactericidal against three different gonococcal strains and interestingly, vaginal secretions were growth inhibitory when human transferrin was provided as a sole iron source in vitro. These studies suggest that the Tbps can elicit antibodies with important and potentially protective biological properties. We are currently testing whether Tbp-specific antibodies are protective in a mouse model of lower female genital tract colonization.

\section{CONCLUSIONS}

TonB dependent transporters enable the efficient use of iron sources that are otherwise unavailable due to insolubility, low concentration, or chelation by iron-binding proteins. N. gonorrhoeae has the capacity to encode up to eight transporters, facilitating the use of a variety of iron compounds derived from or produced in the human host. The genes encoding the lactoferrin-iron acquisition system are intact only in a minority of gonococcal strains, suggesting that selective pressures in vivo have resulted in the loss of lactoferrin binding capability over time. The hemoglobin-iron acquisition system is off, due to phase variation, in most gonococcal strains. However, use of hemoglobin-derived iron through expression of this system seems to be selected for in vivo in women during the early phase of the menstrual cycle. The enterobactinand salmochelin-iron acquisition system is also subject to phase variation, but expression is tuned up or down by alteration of promoter strength, suggesting that the presence of xenosiderophores in the human genital tract could select for variants with enhanced capability to internalize these iron chelates. Substrates and gene expression characteristics have not been defined for the four recently recognized TonB-dependent transporters (TdfF, G, $\mathrm{H}$, and $\mathrm{J}$ ), the genes for which are found in all gonococcal strains sequenced to date. While the substrate has not yet been defined, TdfF appears to play an important role in gonococcal survival inside of human cervical epithelial cells. The transferrin-iron acquisition system is ubiquitously expressed by all gonococcal isolates and is 
the best characterized of all the gonococcal TonB-dependent transport systems. Cumulatively, studies of this iron transport system lead to the following model of vectorial transport of iron from human transferrin to the periplasm (Figure 3). The gonococcal TonB-dependent transporter, TbpA, is sufficient for iron acquisition from transferrin but is aided in this process by the lipoprotein, TbpB. Both proteins bind human transferrin specifically but TbpB further differentiates between the ferrated and apo forms of the protein, allowing for selection of the proper iron-loaded ligand and efficient release of the used transferrin subsequent to iron extraction. Both TbpA and TbpB participate in iron extraction at the cell surface, which results in free iron being captured by the TbpA plug domain. This interaction presumably effects the exposure of the TonB-box domain of TbpA, signaling to TonB that ligand, in this case free iron, is bound and ready for transport. TonB, in its energized form, tugs on the plug domain, causing a localized rearrangement and presentation of the iron atom at the inner orifice

\section{REFERENCES}

Agarwal, S., King, C. A., Klein, E. K., Soper, D. E., Rice, P. A., Wetzler, L. M., and Genco, C. A. (2005). The gonococcal fur-regulated $t b p A$ and $t b p B$ genes are expressed during natural mucosal gonococcal infection. Infect. Immun. 73, 4281-4287.

Anderson, J. E., Hobbs, M. M., Biswas, G. D., and Sparling, P.F. (2003). Opposing selective forces for expression of the gonococcal lactoferrin receptor. $\mathrm{Mol}$. Microbiol. 48, 1325-1337.

Anderson, J. E., Leonie, P. A., Miller, W. C., Chen, C. J., Hobbs, M. M., and Sparling, P. F. (2001). Selection for expression of the gonococcal hemoglobin receptor during menses. J. Infect. Dis. 184, 1621-1623.

Anderson, J. E., Sparling, P. F., and Cornelissen, C.N. (1994). Gonococcal transferrin-binding protein 2 facilitates but is not essential for transferrin utilization. J. Bacteriol. 176, 3162-3170.

Beucher, M., and Sparling, P. F. (1995). Cloning, sequencing, and characterization of the gene encoding FrpB, a major iron-regulated, outer membrane protein of Neisseria gonorrhoeae. J. Bacteriol. 177, 2041-2049.

Biswas, G. D., Anderson, J. E., Chen, C.-J., Cornelissen, C. N., and Sparling, P. F. (1999). Identification and functional characterization of the Neisseria gonorrhoeae $l b p B$ gene product. Infect. Immun. 67, 455-459.

Biswas, G. D., Anderson, J. E., and Sparling, P. F. (1997). Cloning and functional characterization of Neisseria gonorrhoeae tonB, exbB and exbD genes. Mol. Microbiol. 24, 169-179.

Biswas, G. D., and Sparling, P. F. (1995). Characterization of $l b p A$, the structural gene for a lactoferrin receptor in
Neisseria gonorrhoeae. Infect. Immun. 63, 2958-2967.

Boulton, I. C., Yost, M. K., Anderson, J. E., and Cornelissen, C. N. (2000). Identification of discrete domains protein A that are necessary for ligand binding and iron uptake functions. Infect. Immun. 68, 6988-6996.

Buchanan, S. K., Smith, B. S., Venkatramani, L., Xia, D., Esser, L., Palnitkar, M., Chakraborty, R., van der Helm, D., and Deisenhofer, J. (1999). Crystal structure of the outer membrane active transporter FepA from Escherichia coli. Nat. Struct. Biol. 6, 56-63.

Cadieux, N., Bradbeer, C., and Kadner, R. J. (2000). Sequence changes in the Ton box of BtuB affect its transport activities and interaction with TonB protein. J. Bacteriol. 182, 5954-5961.

Carson, S. D., Stone, B., Beucher, M., Fu, J., and Sparling, P. F. (2000). Phase variation of the gonococcal siderophore receptor FetA. Mol. Microbiol. 36, 585-593.

Carson, S. D. B., Klebba, P. E., Newton, S. M. C., and Sparling, P. F. (1999). Ferric enterobactin binding and utilization by Neisseria gonorrhoeae. J. Bacteriol. 181, 2895-2901.

Chen, C. J., Elkins, C., and Sparling, P. F. (1998). Phase variation of hemoglobin utilization in Neisseria gonorrhoeae. Infect. Immun. 66, 987-993.

Chen, C. J., Mclean, D., Thomas, C. E. Anderson, J. E., and Sparling, P. F. (2002). Point mutations in $\mathrm{HpuB}$ enable gonococcal HpuA deletion mutants to grow on hemoglobin. J. Bacteriol. 184, 420-426.

Chen, C.-J., Sparling, P. F., Lewis, L. A., Dyer, D. W., and Elkins, C. (1996). Identification and purification of a hemoglobin-binding outer membrane within gonococcal transferrin-binding

of the barrel, whereupon FbpA sequesters the iron for transport across the periplasm. Because expression of the transferrin-iron acquisition system is conserved among strains and necessary to initiate human male infection, the proteins that comprise this system are considered viable vaccine candidates. Initial studies suggest that vaccination with these proteins elicits biologically functional antibodies engendering hope that a gonococcal vaccine containing these proteins (and perhaps others too) could be efficacious and protective against the common STI caused by N. gonorrhoeae.

\section{ACKNOWLEDGMENTS}

Funding for this work was provided by U.S. Public Health Service grant numbers AI047141, AI065555, and AI084400 from the National Institute of Allergy and Infectious Diseases at the National Institutes of Health. Cynthia Nau Cornelissen is also supported by the SE STI Center grant (U19 AI31496) from the National Institute of Allergy and Infectious Diseases.

protein from Neisseria gonorrhoeae. Infect. Immun. 64, 5008-5014.

Chen, C.-Y., Berish, S. A., Morse, S. A. and Meitzner, T. A. (1993). The ferric iron-binding protein of pathogenic Neisseria spp. functions as a periplasmic transport protein in iron acquisition from human transferrin. $\mathrm{Mol}$. Microbiol. 10, 311-318.

Connell, T. D., Shaffer, D., and Cannon, J. D. (1990). Characterization of the repertoire of hypervariable regions in the Protein II (opa) gene family of Neisseria gonorrhoeae. Mol. Microbiol. 4, 439-449.

Cornelissen, C. N., Anderson, J. E., and Sparling, P. F. (1997a). Characterization of the diversity and the transferrin-binding domain of gonococcal transferrin-binding protein 2. Infect. Immun. 65, 822-828.

Cornelissen, C. N., Anderson, J. E., and Sparling, P. F. (1997b). Energydependent changes in the gonococcal transferrin receptor. Mol. Microbiol. 26, 25-35.

Cornelissen, C. N., Anderson, J. E. Boulton, I. C., and Sparling, P. F. (2000). Antigenic and sequence diversity in gonococcal transferrin-binding protein A (TbpA). Infect. Immun. 68, 4725-4735.

Cornelissen, C. N., Biswas, G. D., and Sparling, P. F. (1993). Expression of gonococcal transferrin-binding protein 1 causes Escherichia coli to bind human transferrin. J. Bacteriol. 175 , 2448-2450.

Cornelissen, C. N., Biswas, G. D., Tsai, J., Paruchuri, D. K., Thompson, S. A., and Sparling, P. F. (1992). Gonococcal transferrin-binding protein 1 is required for transferrin utilization and is homologous to TonB-dependent outer membrane receptors. J. Bacteriol. 174, 5788-5797.
Cornelissen, C. N., Kelley, M., Hobbs, M. M., Anderson, J. E., Cannon, J. G., Cohen, M. S., and Sparling, P.F. (1998). The transferrin receptor expressed by gonococcal strain FA1090 is required for the experimental infection of human male volunteers. $\mathrm{Mol}$. Microbiol. 27, 611-616.

Cornelissen, C. N., and Sparling, P. F. (1996). Binding and surface exposure characteristics of the gonococcal transferrin receptor are dependent on both transferrin-binding proteins. $J$ Bacteriol. 178, 1437-1444.

DeRocco, A. J., and Cornelissen, C. N. (2007). Identification of transferrinbinding domains in TbpB expressed by Neisseria gonorrhoeae. Infect. Immun. 75, 3220-3232.

DeRocco, A. J., Yost-Daljev, M. K., Kenney, C. D., and Cornelissen, C. N. (2008). Kinetic analysis of ligand interaction with the gonococcal transferrin-iron acquisition system. Biometals 22, 439-451.

Dhungana, S., Taboy, C. H., Anderson, D. S., Vaughan, K. G., Aisen, P., Mietzner, T.A., and Crumbliss, A. L. (2003). The influence of the synergistic anion on iron chelation by ferric binding protein, a bacterial transferrin. Proc. Natl. Acad. Sci. U.S.A. 100, 3659-3664

Ducey, T. F., Carson, M. B., Orvis, J., Stintzi, A. P., and Dyer, D. W. (2005). Identification of the iron-responsive genes of Neisseria gonorrhoeae by microarray analysis in defined medium. J. Bacteriol. 187, 4865-4874.

Dyer, D. W., West, E. P., and Sparling, P. F. (1987). Effects of serum carrier proteins on the growth of pathogenic Neisseriae with heme-bound iron. Infect. Immun. 55, 2171-2175.

Escolar, L., Perez-Martin, J., and de Lorenzo, V. (1999). Opening the iron box: transcriptional metalloregulation 
by the Fur protein. J. Bacteriol. 181, 6223-6229.

Folster, J. P., and Shafer, W. M. (2005). Regulation of $m t r F$ expression in Neisseria gonorrhoeae and its role in high-level antimicrobial resistance. J. Bacteriol. 187, 3713-3720.

Gumbart, J., Wiener, M. C., and Tajkhorshid, E. (2007). Mechanics of force propagation in TonB-dependent outer membrane transport. Biophys. J. 93, 496-504.

Hagen, T. A., and Cornelissen, C. N. (2006). Neisseriagonorrhoeae requires expression of TonB and the putative transporter TdfF to replicate within cervical epithelial cells. Mol. Microbiol. 62, 1144-1157.

Hamilton, H., Dominguez, N., Schwartz, K., Hackett, K., and Dillard, J. (2005). Neisseria gonorrhoeae secrete chromosomal DNA via a novel type IV secretion system. Mol. Microbiol. 55, 1704-1721.

Hantke, K., Nicholson, G., Rabsch, W., and Winkelmann, G. (2003).Salmochelins, siderophores of Salmonella enterica and uropathogenic Escherichia coli strains, are recognized by the outer membrane receptor iron. Proc. Natl. Acad. Sci. U.S.A. 100, 3677-3682.

Hook, E. W. I., and Handsfield, H. H. (2008). "Gonococcal infections in the adult," in Sexually Transmitted Diseases, 4th Edn, eds K. K. Holmes, P. F. Sparling, W. E. Stamm, P. Piot, J. N. Wasserheit, L. Corey, M. S. Cohen, and D. H. Watts (New York: McGrawHill), 31-52.

Jackson, L. A., Ducey, T. F., Day, M. W., Zaitshik, J. B., Orvis, J., and Dyer, D. W. (2010). Transcriptional and functional analysis of the Neisseria gonorrhoeae Fur regulon. J. Bacteriol. 192, 77-85.

Jerse, A. (1999). Experimental gonococcal genital tract infection and opacity protein expression in estradiol-treated mice. Infect. Immun. 67, 5699-5708.

Jerse, A. E., Cohen, M. S., Drown, P. M., Whicker, L. G., Isbey, S. F., Seifert, H. S., and Cannon, J. G. (1994). Multiple gonococcal opacity proteins are expressed during experimental urethral infection in the male. J. Exp. Med. 179, 911-920.

Jerse, A. E., Crow, E. T., Bordner, A. N., Rahman, I., Cornelissen, C. N., Moench, T. R., and Mehrazar, K. (2002). Growth of Neisseria gonorrhoeae in the female mouse genital tract does not require the gonococcal transferrin or hemoglobin receptors and may be enhanced by commensal lactobacilli. Infect. Immun. 70, 2549-2558.

Kenney, C. D. (2002). Characterization of the transferrin-binding protein complex expressed by Neisseria gonorrhoeae. Microbiol. Immunol. Virginia Commonwealth University.

Kenney, C. D., and Cornelissen, C. N. (2002). Demonstration and characterization of a specific interaction between gonococcal transferrin binding protein A and TonB. J. Bacteriol. 184, 6138-6145.

Kohlhoff, S. A., and Hammerschlag, M. R. (2008). "Gonococcal and chlamydial infections in infants and children," in Sexually Transmitted Diseases, 4th Edn, eds K. K. Holmes, P. F. Sparling, W. E. Stamm, P. Piot, J. N. Wasserheit, L. Corey, M. S. Cohen, and D. H. Watts (New York: McGraw Hill), 629-639.

Larson, J. A., Higashi, D. L., Stojiljkovic, I., and So, M. (2002). Replication of Neisseria meningitidis within epithelial cells requires TonB-dependent acquitision of host cell iron. Infect. Immun. 70, 1461-1467.

Marri, P. R., Paniscus, M., Weyand, N. J., Rendon, M. A., Calton, C. M., Hernandez, D. R., Higashi, D. L., Sodergren, E., Weinstock, G. M., Rounsley, S. D., and So, M. (2010). Genome sequencing reveals widespread virulence gene exchange among human Neisseria species. PLoS ONE, 5, e11835. doi: 10.1371/journal. pone. 0011835

Masri, H. (2003). Function and immunogenicity of individual epitopes of gonococcal transferrin binding protein A. Microbiol. Immunol. Virginia Commonwealth University.

Masri, H. P., and Cornelissen, C. N. (2002). Specific ligand binding attributable to individual epitopes of gonococcal transferrin binding protein A. Infect. Immun. 70, 732-740.

McKenna, W. R., Mickelsen, P.A.,Sparling, P. F., and Dyer, D. W. (1988). Iron uptake from lactoferrin and transferrin by Neisseria gonorrhoeae. Infect. Immun. 56, 785-791.

Mickelsen, P. A., Blackman, E., and Sparling, P. F. (1982). Ability of Neisseria gonorrhoeae, Neisseria meningitidis, and commensal Neisseria species to obtain iron from lactoferrin. Infect. Immun. 35, 915-920.

Mickelsen, P.A., and Sparling, P. F. (1981). Ability of Neisseria gonorrhoeae, Neisseria meningitidis, and commensal Neisseria species to obtain iron from transferrin and iron compounds. Infect. Immun. 33, 555-564.

Moraes, T. F., Yu, R. H., Strynadka, N. C., and Schryvers, A. B. (2009). Insights into the bacterial transferrin receptor: the structure of transferrin-binding protein B from Actinobacillus pleuropneumoniae. Mol. Cell 35, 523-33.

Noinaj, N., Guillier, M., Barnard, T. J., and Buchanan, S. K. (2011). TonBdependent transporters: regulation, structure, and function. Annu. Rev. Microbiol. 64, 43-60.

Noto, J., and Cornelissen, C. N. (2008) Identification of $\mathrm{TbpA}$ residues required for transferrin-iron utilization by Neisseria gonorrhoeae. Infect. Immun. 76, 1960-1969.

Nowalk, A. J., Tencza, S. B., and Mietzner, T. A. (1994). Coordination of iron by ferric iron-binding protein of pathogenic Neisseria is homologous to the transferrins. Biochemistry 33 12769-12775.

O'Brien, I. G., and Gibson, F. (1970). The structure of enterochelin and related 2,3-dihydroxy-N-benzoylserine conjugates from Escherichia coli. Biochim. Biophys. Acta 215, 393-402.

Pawelek, P. D., Croteau, N., Ng-ThowHing, C., Khursigara, C.M., Moiseeva, N., Allaire, M., and Coulton, J. W. (2006). Structure of TonB in complex with FhuA, E. coli outer membrane receptor. Science 312, 1399-1402.

Perry, R. D., and San, C., and Clemente, L. (1979). Siderophore synthesis in Klebsiella pneumoniae and Shigella sonnei during iron deficiency. $J$. Bacteriol. 140, 1129-1132.

Pollack, J. R., and Neilands, J. B. (1970). Enterobactin, an iron transport compound from Salmonella typhimurium Biochem. Biophys. Res. Commun. 38 989-992.

Postle, K., and Larsen, R. A. (2007) TonB-dependent energy transduction between outer and cytoplasmic membranes. Biometals 20, 453-465.

Price, G.A.,Hobbs,M.M., and Cornelissen, C. N. (2004). Immunogenicity of gonococcal transferrin binding proteins during natural infections. Infect. Immun. 72, 277-283.

Price, G. A., Masri, H. P., Hollander, A. M., Russell, M. W., and Cornelissen, C. N (2007). Gonococcal transferrin binding protein chimeras induce bactericidal and growth inhibitory antibodies in mice. Vaccine 25, 7247-7260.

Price, G. A., Russell, M. W., and Cornelissen, C. N. (2005). Intranasal administration of recombinant Neisseria gonorrhoeae transferrin binding proteins $\mathrm{A}$ and $\mathrm{B}$ conjugated to the cholera toxin $B$ subunit induces systemic and vaginal antibodies in mice. Infect. Immun. 73, 3945-3953.

Ronpirin, C., Jerse, A. E., and Cornelissen, C. N. (2001). The gonococcal genes encoding transferrin binding proteins (Tbp) A and B are arranged in a bicistronic operon but are subject to differential expression. Infect. Immun. 69, 6336-6347.

Schneider, M. C., Prosser, B. E., Caesar, J. J., Kugelberg, E., Li, S., Zhang, Q. Quoraishi, S., Lovett, J. E., Deane, J. E., Sim, R. B., Roversi, P., Johnson, S.,
Tang, C. M., and Lea, S. M. (2009). Neisseria meningitidis recruits factor $\mathrm{H}$ using protein mimicry of host carbohydrates. Nature 458, 890-893.

Sebastian, S., Agarwal, S., Murphy, J. R., and Genco, C. A. (2002). The gonococcal fur regulon: identification of additional genes involved in major catabolic, recombination, and secretory pathways. J. Bacteriol. 184, 3965-3974. Sebastian, S., and Genco, C. A. (1999). $\mathrm{FbpC}$ is not essental for iron acquisition in Neisseria gonorrhoeae. Infect. Immun. 67, 3141-3145.

Shultis, D. D., Purdy, M. D., Banchs, C. N., and Wiener, M. C. (2006). Outer membrane active transport: structure of the BtuB:TonB complex. Science 312, 1396-1399.

Siburt, C. J. P., Roulhac, P. L., Weaver K. D., Noto, J. M., Mietzner, T. A., Cornelissen, C. N., Fitzgerald, M. C. and Crumbliss, A. L. (2009). Hijacking transferrin bound iron: protein-receptor interactions involved in iron transport in N. gonorrhoeae. Metallomics 1 , 249-255.

Snyder, L. A., and Saunders, N. J. (2006). The majority of genes in the pathogenic Neisseria species are present in non-pathogenic Neisseria lactamica including those designated as 'virulence genes'. BMC Genomics 7, 128. doi: 10.1186/1471-2164-7-129

Stojiljkovic, I., Larson, J., Hwa, V., Anic, S., and So, M. (1996). HmbR outer membrane proteins of pathogenic Neisseriae: iron-regulated, hemoglobin binding proteins with high degree of primary structure conservation. J. Bacteriol. 178, 3341-3352.

Stork, M., Bos, M. P., Jongerius, I., de Kok, N., Schilders, I., Weynants, V. E., Poolman, J. T., and Tommassen, J. (2011). An outer membrane receptor of Neisseria meningitidis involved in zinc acquisition with vaccine potential. PLoS Pathog. 6, e1000969. doi: 10.1371/journal.ppat.1000969

Strange, H. R., Zola, T.A., and Cornelissen, C. N. (2011). The $f b p A B C$ operon is required for Ton-independent utilization of xenosiderophores by Neisseria gonorrhoeae strain FA19. Infect. Immun. 79, 267-278.

Taboy, C. H., Vaughan, K. G., Mietzner, T. A., Aisen, P., and Crumbliss, A. L. (2001). $\mathrm{Fe}^{3+}$ coordination and redox properties of a bacterial transferrin. J. Biol. Chem. 276, 2719-2724.

Thomas, C. E., and Sparling, P. F. (1996). Isolation and analysis of a fur mutant of Neisseria gonorrhoeae. J. Bacteriol. 178, 4224-4232.

Turner, P. C., Thomas, C. E., Stojiljkovic, I., Elkins, C., Kizel, G., Ala'Aldeen, D. A., and Sparling, P. F. (2001). Neisserial Ton B-dependent 
outer-membrane proteins: detection, regulation and distribution of three putative candidates identified from the genome sequences. Microbiology 147, 1277-1290.

Wally, J., and Buchanan, S. K. (2007). A structural comparison of human serum transferrin and human lactoferrin. Biometals 20, 249-262.

West, S. E. H., and Sparling, P. F. (1985). Response of Neisseria gonorrhoeae to iron limitation: alterations in expression of membrane proteins without apparent siderphore production. Infect. Immun. 47, 388-394.
West, S. E. H., and Sparling, P. F. (1987). Aerobactin utilization by Neisseria gonorrhoeae and cloning of a genomic DNA fragment that complements Escherichia coli fhuB mutations. J. Bacteriol. 169, 3414-3421.

Yost-Daljev, M. K., and Cornelissen, C. N. (2004). Determination of surfaceexposed, functional domains of gonococcal transferrin-binding protein A. Infect. Immun. 72, 1775-1785.

Zola, T. A., Strange, H. R., Dominguez, N. M., Dillard, J. P., and Cornelissen, C. N. (2010). Type IV secretion machinery promotes ton-independent intracellular survival of Neisseria gonorrhoeae within cervical epithelial cells. Infect. Immun. 78, 2429-2437.

Conflict of Interest Statement: The authors declare that the research was conducted in the absence of any commercial or financial relationships that could be construed as a potential conflict of interest.

Received: 21 March 2011; accepted: 11 May 2011; published online: 27 May 2011.

Citation: Cornelissen $\mathrm{CN}$ and Hollander A (2011) TonB-dependent transporters expressed by Neisseria gonorrhoeae. Front. Microbio. 2:117. doi: 10.3389/ fmicb.2011.00117

This article was submitted to Frontiers in Cellular and Infection Microbiology, a specialty of Frontiers in Microbiology.

Copyright (c) 2011 Cornelissen and Hollander. This is an open-access article subject to a non-exclusive license between the authors and Frontiers Media $S A$, which permits use, distribution and reproduction in other forums, provided the original authors and source are credited and other Frontiers conditions are complied with. 